\title{
Food Traceability for Smart Agriculture Through Block-chain
}

\author{
Anjali Mehta ${ }^{1}$, Dr. Priyanka Sharma ${ }^{1}$ \\ ${ }^{1}$ School of Information Technology, Artificial Intelligence, and Cyber Security, Rashtiya Raksha University, \\ Gandhinagar, Gujarat, India
}

\section{ABSTRACT}

\section{Article Info}

Volume 8, Issue 3

Page Number: 129-134

\section{Publication Issue :}

May-June-2021

\section{Article History}

Accepted : 12 May 2021

Published: 22 May 2021
Blockchain is an emerging digital technology that allows for widespread financial transactions between underutilized groups, without the need for intermediaries such as banks. The report looks at why blockchain is better than the current traditional system, and discusses the effects, challenges and potential of overall asset management. Complete research shows that blockchain is a promising technology aimed at transparent food marketing, with many ongoing programs for various food products and food-related problems, but many challenges and challenges remain, preventing its widespread popularity among farmers and programs.

Keywords : Blockchain, Food Tracking, Smart Agriculture

\section{INTRODUCTION}

About twenty-first century technology. As the need for modernization in our continual life grows, people are accepting new progress. From the use of the remote for customizable gadgets, to the misuse of voice notes to place orders, there have been innovations in use in our traditional life. Advances such as augmented reality and IoT have gained momentum over the past decade and are now a new expansion of new plastics in blockchain technology packs, for example. The blockchain-based supply chain is reliable and trustworthy in identification and authentication, which also eliminates middleman auditors. Implementing a blockchain to follow all the activities in the listed network is one of the main potential activities, for example, who is playing the activity, at what time and where the area of each activity. Therefore, blockchain can give greater assurance that things are real and outstanding, also reduce costs and expand efficiency. The main objective of this research work is to create a decentralized, democratic, transparent "food discovery platform" in the supply chain based on blockchain technology.

\section{PROBLEM DESCRIPTION}

This research is based not only on the impact on the environment, but also on its economic impact on farmers, distributors, food processing people, retailers and customers, on a viable major challenge to sustainable agriculture. In the current system for the supply of food and crops there is an "intermediary" between each shareholder. The presence of intermediaries increases the cost and the shareholders are unable to get the value of the crop / food. There is currently no automated system for obtaining crop values based on money transactions, or its quality or the ingredients used to make food products. In the 
current system, manual disposal of bills (money) and paperwork is very necessary to transfer ownership. There are also some issues of blockchain such as scalability, delay issues, low transaction speed, regularity, blockchain acceptability. The main drawbacks are the high consumption of hardware, the energy and time required for the mining process, even the fact that the technology is complex and difficult to understand. There are also many blockchain platforms that rely on different consensus algorithms, so it can be very difficult to choose the right platform for the development of a supply food chain.

\section{OBJECTIVES}

1. To get complete traceability of food/product.

2. The farmers/food producer gets the satisfactory price.

3. Customer/Users can inspect the quality of food/product.

4. Automated pricing based on the quality of food.

\section{LITERATURE REVIEW}

Some important projects that use blockchain technology based on effects, partners, security, security, etc.: For example, Walmart is using blockchain to follow offensive vulnerabilities. In fact, the ability to identify the root cause of food-diseases is probably not the most misused of innovation in nutritional frameworks. Another fruit that used Walmart blockchain technology is for mangoes. The result of the IBM and Walmart project is a single historical record of all the stages involved in the general production, processing and distribution systems. This includes tracking the mango crop in Mexico through Walmart product sales in the United States. It took a week for mangoes to be tracked using their current processes, a blockchain approach initiated by Walmart that allowed executives to determine the origin of mango production in just 2 seconds. Grassroots Farmers Cooperative (Grassroots
Farmers Aggregate 2017) sells a meat subscription box that provides shoppers with a concrete awareness of the growing status of their organisms using blockchain innovation. In the demonstrated pilot, examples of chicken assigned in San Francisco were put together with $\mathrm{QR}$ codes that link to their meat story. Consumers will have the opportunity to check the $\mathrm{QR}$ code on the Grass Roots item to see where the meat originated and how the organisms grew. The "advanced history" of meat includes stories about people involved in the making of the final product from ranches to butchers. One of Ripe.io's first attacks was Tomato's Internet, a community-based venture of analog devices, and a quick easy plate installation of SweetGreen's mixed celery. With the Tomato Internet, Ripe.yo has ordered a plethora of information on how to use the blockchain innovation and apply it to grow good tomatoes. Using ranchers and sensor machines, it collects data on everything from temperature to humidity, to tomato science study. Through the services provided by the Origin Trail Company, consumers can see from which plantations the material they grow has grown, the source and growing condition of the poultry and so on. In addition, the "Blockchain for Agri-Food" project developed a proof-of-concept. Blockchain based application about table grapes from South Africa. A framework has been proposed for greenhouse farming with improved security based on blockchain technology

Supply chain management involves the implementation of various processes in the supply chain along with a comprehensive plan. Some problems in the current system can be solved by the supply chain.

Problem 1: It is difficult to confirm that most materials and components come from Asian markets such as China. As a consumer, you never really know the true value of the products you are using. Also, the lack of transparency in modern supply chains is another major problem. If your phone has a defective 
part, it is impossible to pinpoint exactly where that defective part came from and who is responsible for it. In fact, let me give you an example of how dangerous a lack of transparency can really be. On October 6, 2006, several US states were hit by a major e-coli outbreak. Criminal? Spinach - Approximately 199 people were affected, 22 of whom were children under 5 years of age. In 199, 31 people developed renal failure called hemolytic-uremic syndrome and 3 died.

As a result, the entire food industry fell into crisis. People are desperately trying to identify the source of the infected spinach. Everyone immediately stopped selling spinach from the market. It took the Food and Drug Administration (FDA) a total of 2 weeks to determine the source of the contaminated spinach. What is the source Can you back up? It is the supplier. A farm. A pile. One farm closed the entire industry for 2 weeks. By that time, the farmers who depended on spinach for their livelihood had broken up and become impoverished. All of these can be avoided if there is a good way to identify and track defective spinach. It can be provided through the food chainability platform through blockchain.

\section{Problem 2: Corruption:}

Supply Somewhere in your supply chain, someone is caught wanting to get a product from a specific supplier, without looking at other offers. When confronted, they emphasize that only that supplier can meet certain specifics, which may or may not be true.

Company Suppose a company expands its product range. The required components can be obtained from a specific supplier, however, the supplier also has a role to play.

Supply Some of your collection executives prefer certain suppliers in your supply chain because of personal relationships.

Product Suppose you need a new component /material for a new product. However, that material is a rare asset, and only one supplier can supply it to you.
One of your collection officers is running a special contract with your supplier. Instead of a bribe, the supplier can replace the quality of its products.

Main One of your suppliers secretly assigns work to sub-suppliers who do not adhere to the code of conduct that you impress on your major suppliers.

A supplier delivers the item on time and in good condition, but claims additional costs and additional costs, forcing the contract to be re-negotiated in the process.

You simply have way too many components in your supply chain to keep track of them all. As such, suppliers can pretty much get away with anything. This is a big problem that traditional supply chain management systems have. They are not scalable.

\section{Problem 3: Costs:}

The cost factor can be divided among four types of different cost occurring in the supply chain such as :

\section{- Procurement Costs}

- Transportation Costs

- Inventory Costs

- Quality Costs

\section{Problem 4: Globalization:}

Globalization means that your company becomes internationally influential or large enough to start operating internationally. As you can see, globalization opens up many challenges for supply chain management. Most companies have to run their supply chains through multiple countries to buy different parts of their products. However, it brings a lot of problems. Keep in mind that your suppliers are located in very different geographical locations, which makes coordination and cooperation really difficult. In other words, it's really hard to know if they're really doing their job. Another issue that comes into the picture for blockchain technology is choosing the right platform for your project. There are many platforms in blockchain

1. Ethereum

2. Hyperledger Fabric 
3. Hyperledger Sawtooth

4. Hedera Hashgraph

5. Ripple

6. Quorum

\section{PROPOSED SYSTEM}

Our ecosystem involves the traditional ERP (Enterprise Resource Planning) legacy system and a new IoT system. Farm companies, farming processing plants, plantation companies, planting processing plants, logistics companies and food retail storefronts as well as the customers can use their smart mobile phone as a portal or blockchain thin node to access the data stored in the chain. The core of the whole architecture is a virtual Trusted Trade Blockchain Network Cloud Platform.

\section{A. Tools and technologies}

\section{- Ethereum :}

Ethereum is a distributed public blockchain network focused on implementing the programming code of any decentralized application. More simply, it is a platform for sharing information around the world, which is immutable or unchangeable. The main feature of Ethereum is that it is highly extensible, which is a programmable blockchain. This is equivalent to a programmable distributed ledger, where everyone agrees to run the same program with the same data. It's not just bookkeeping, it's a global, shared data processing protocol. Hence, the global computer idea. Major Ethereum blockchain access is limited, meaning anyone can join the network. There are some exceptions to this, but most importantly, you can participate as long as you have an internet connection and follow the protocol. Each participant running the protocol performs and records the node in the network and the same activity. An action on a network is called a transaction. The transaction is divided into blocks. Only one block is added at a time and mathematical proofs confirm the order and authenticity of the blocks. It syncs the 'distributed spreadsheet. Every participant in the network processes every transaction, resulting in the system being expensive to maintain and modify. In order to reduce spam and discourage worthless transactions, gas is consumed for every operation in the network. Gas is the fuel of the Ethereum network. Blockchain needs any modification. Customers can choose how much to pay for gas per transaction - the more they are willing to pay, the faster their transactions will be processed through the network.

\section{- Ganache:}

Ganache allows you to create a private etherium blockchain to run tests, execute commands and monitor the state while controlling the operation of the chain. It gives you the ability to do all the things you do in the main chain without cost. Many developers use it to test their smart contracts during development. It offers convenient tools such as advanced mining control and built-in block explorer. After we create the workspace, the screen shows some details about the server and also lists several accounts. Each account is given 100 ethers. You can focus on developing your application by having Ether automatically in all accounts.

\section{- Truffle:}

Truffle is a property pipeline for developer environment, test framework and blockchain. It allows developers to spin a smart contract project at the click of a button and provides you with project structure, files and directories that make extension and testing much easier (otherwise you need to configure them). As a developer, you create a truffle project that demonstrates the structure of your project. Once you start coding a bit, you may want to test the code, but it requires a blockchain. Now you are running the computer to become this blockchain. In your Deployment file (the file that Truffle gives you when creating a project), you can specify whether your project should use Ganachi or use the 
main network. You can run Truffle Migrate (runs Truffle compilation automatically for you) to execute agreements with the data provided in the migration files.

\section{SYSTEM DESIGN}

\section{A. System Architecture:}

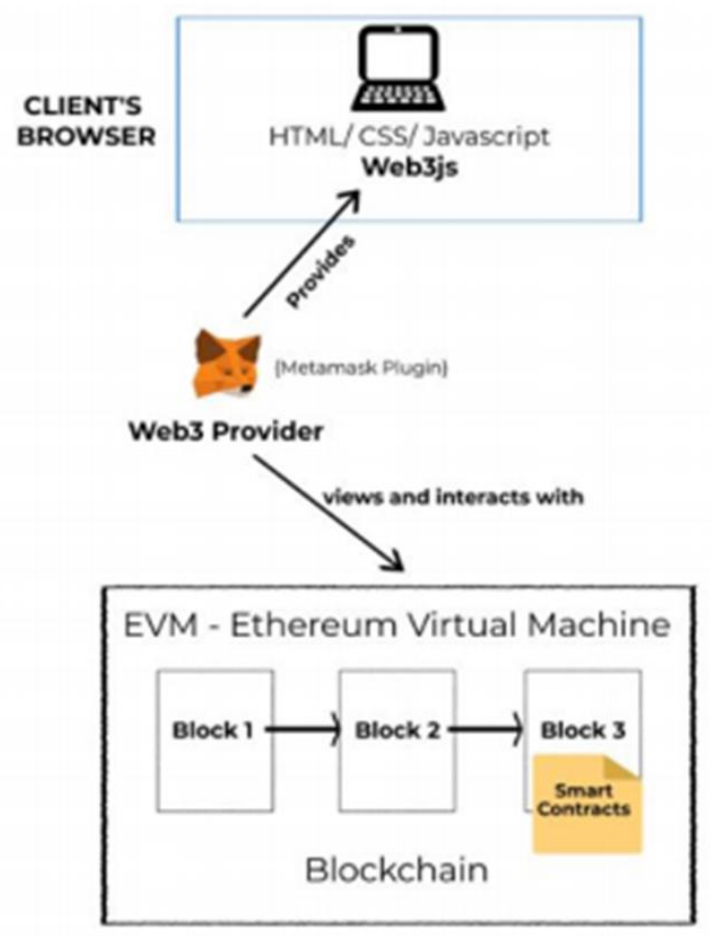

Fig.1: Architecture Design

\section{B. Data Flow Diagram:}

There are two types of transaction data that will be stored in the blockchain, as shown in Fig. 2: one is that generated from traditional ERP legacy systems, such as the trade, logistics, delivery, warehousing information etc.; another is that generated from IoT devices, such as the air temperature, air humidity, soil $\mathrm{PH}$, soil nutrition, ground moisture data etc. After hashing and digital signing, those data will be sent to the entire nodes of blockchain system directly or through the IoT gateways, where they will be verified, added into transaction pool, and stored into blockchain.

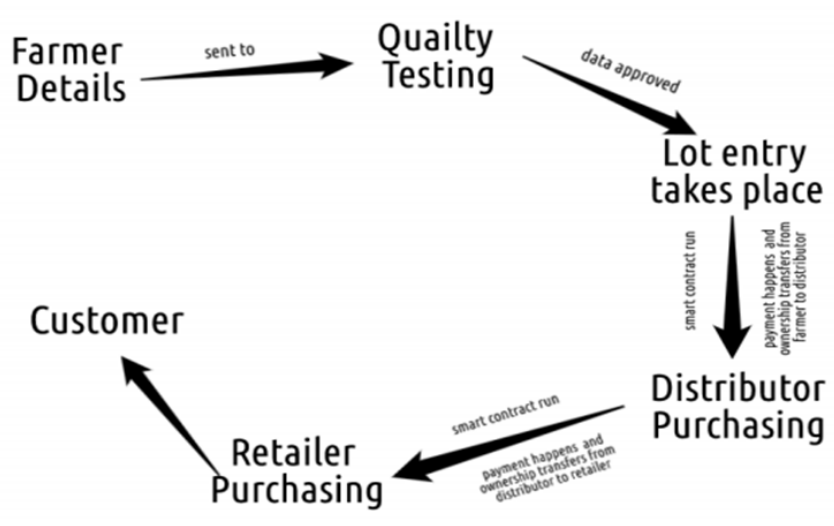

Fig.2: System Flow

\section{EXPECTED OUTCOME}

Customers can use their computers or mobile phones to retrieve and verify all transaction data. For example, a buyer buys a box of milk from a supermarket and then retrieves all related transaction data, including milk, using a smartphone to scan the 2-D barcode. Produced from any farm, any day and time of production, cow ID on the farm, ID of milk collecting workers, equipment information collection, packaging information, milk production, process, logistics, all temperature and other environmental data for storage, etc. blockchain system Can be verified all information.

\section{CONCLUSION}

As such, blockchain technology is already being used through a number of projects and programs, aiming to create a proven and reliable environment for building transparent and more sustainable food production and distribution, bringing shareholders into the supply chain. However, there are still many issues and challenges that need to be addressed beyond the technical level. To reduce barriers to use, governments should set an example and promote the digitization of public administration. They need to invest more in education and training as well as research and innovation to present and present evidence for the potential benefits of this technology. 


\section{REFERENCES}

[1]. The rise of blockchain technology in agriculture and food supply chains: Andreas Kamilarisa, Agusti Fontsa, Francesc X. Prenafeta-Boldúa

[2]. Transparency in food supply chains: A review of enabling technology solutions: Jake Astilla, Rozita A. Darab, Malcolm Campbellc, Jeffrey M. Farberd, Evan D.G. Frasere,Shayan Sharifa, Rickey Y. Yadaf

[3]. Understanding Blockchain Opportunities and Challenge: Carmen HOLOTESCU

[4]. Blockchain challenges and opportunities: a survey by Zibin Zheng and Shaoan Xie, HongNing Dai, Xiangping Chen, Huaimin Wang

[5]. Blockchains for Supply Chain Management: Architectural Elements and Challenges Towards a Global Scale Deployment: Antonios Litke , Dimosthenis Anagnostopoulos and Theodora Varvarigou

[6]. Blockchains in operations and supply chains: A model and reference implementation Petri Helo, Yuqiuge Hao

[7]. https://hackr.io/blog/blockchain-programmingbeginners-guide

[8]. https://blockgeeks.com/guides/blockchain-andsupply-chain/\#Quality_Costs

[9]. https://hackernoon.com/how-is-blockchaindisrupting-the-supply-chainindustryf3a1c599daef

[10]. https://openledger.info/insights/blockchain-inthe-supply-chain-use-casesexamples/

\section{Cite this Article}

Anjali Mehta, Dr. Priyanka Sharma "Food Traceability for Smart Agriculture Through Blockchain", International Journal of Scientific Research in Science, Engineering and Technology (IJSRSET), Online ISSN : 2394-4099, Print ISSN : 2395-1990, Volume 8 Issue 3, pp. 129-134, May-June 2021. Available at

doi : https://doi.org/10.32628/IJSRSET218320 Journal URL : https://ijsrset.com/IJSRSET218320 